\title{
Clostridium Difficile Pathogenicity Mechanism Associated with Dysbiosis Caused by The Use of Antibacterials
}

\author{
Ana María Valencia Castaño ${ }^{*}$, Gabriela Carvajales Lozano² ${ }^{2}$ Jamer Luis Benítez Ávila $^{3}$, Katherine \\ Stella Humanes Moreno ${ }^{4}$, Enrique Arturo Lombana Salas ${ }^{5}$, Luis Guillermo Ely Noriega ${ }^{4}$, Ángela \\ María Argumedo Castellanos ${ }^{4}$ and Julieth Alejandra Quiroga Orjuela ${ }^{6}$
}

${ }^{1}$ General Physician, Fundación Universitaria San Martín, Colombia

${ }^{2}$ General Physician, Universidad del Norte de Barranquilla, Colombia

${ }^{3}$ General Physician, Corporación Universitaria Rafael Núñez, Colombia

${ }^{4}$ General Physician, Universidad del Sinú, Colombia

${ }^{5}$ General Physician, University of Sucre, Colombia

${ }^{6}$ General Physician, Universidad Pontificia Bolivariana, Colombia

*Corresponding author: Ana María Valencia Castaño, General Medical San Martin University Foundation, Colombia.

To Cite This Article: Ana María Valencia Castaño, Gabriela Carvajales Lozano, Jamer Luis Benítez Ávila, Katherine Stella Humanes Moreno, Enrique Arturo Lombana Salas, Luis Guillermo Ely Noriega, Ángela María Argumedo Castellanos and Julieth Alejandra Quiroga Orjuela. Clostridium Difficile Pathogenicity Mechanism Associated with Dysbiosis Caused by The Use of Antibacterials. Am J Biomed Sci \& Res. 2021 13(6). AJBSR.MS.ID.001938. DOI: 10.34297/AJBSR.2021.13.001938.

Received: 眥 July 06, 2021; Published: 海 August 24, 2021

\begin{abstract}
Clostridium difficile is a gram-positive rod responsible for the majority of hospital-acquired gastrointestinal infections in humans. Recently in the world literature an exponential increase in its incidence has been reported, involving new more virulent strains that affect new groups of patients that were previously unaffected, generating a series of changes in the clinical presentation of the disease and in the treatment, which influences the prognosis of the disease in these patients. An early diagnosis is essential for therapeutic management and for the implementation of containment measures. However, the speed with which new strains with greater pathogenicity are developed is higher than that with which new drugs are developed to combat them, all this as a result of the irrational use of antibiotics that alter the pathogenicity mechanism of this type of bacteria. In this article we review the pathophysiological aspects of $\mathrm{C}$. difficile infection associated with dysbiosis caused by the use of antibacterials, with the aim of contributing to the preparation of general practitioners and specialists to provide timely and quality care to those who receive it. suffer from this disease and make a more rational use of antibiotics.
\end{abstract}

Keywords: Clostridium difficile, Dysbiosis, Antibiotics

\section{Introduction}

Clostridioides difficile, formerly known as difficult Clostridium, is a spore-forming gram-positive anaerobic bacterium. Spores are bacterial structures that allow C. difficile to resist in the environment and spread from infected to healthy individuals. Certain circumstances such as dysbiosis caused by the irrational use of antibiotics favor the germination of spores in the large intestine, where they take a vegetative form that leads to a clinical infection. In the infection phase, C. difficile produces two types of toxins, enterotoxin A and cytotoxin B, which damage colonocytes and trigger the inflammatory response (Figure 1), leading to a variety of clinical pictures, from colitis of mild intensity to a pseudomembranous colitis or a toxic megacolon that can lead to the patient's condition $[1,2]$.

Antibiotic treatment with penicillins, cephalosporins (especially third-generation ones), lincosamides (clindamycin), and fluoroquinolones is an important risk factor for the development 
of C. difficile infection, although theoretically any antibiotic is a potential risk factor for developing it. It affects up to around $10 \%$ of hospitalized patients and is the most common cause of nosocomial diarrhea globally. It is the causative agent in $20-30 \%$ of cases of diarrhea associated with the use of antibiotics, $60-80 \%$ of colitis associated with the use of antibiotics, and $80-100 \%$ of episodes of pseudomembranous colitis associated with antibiotics [3].

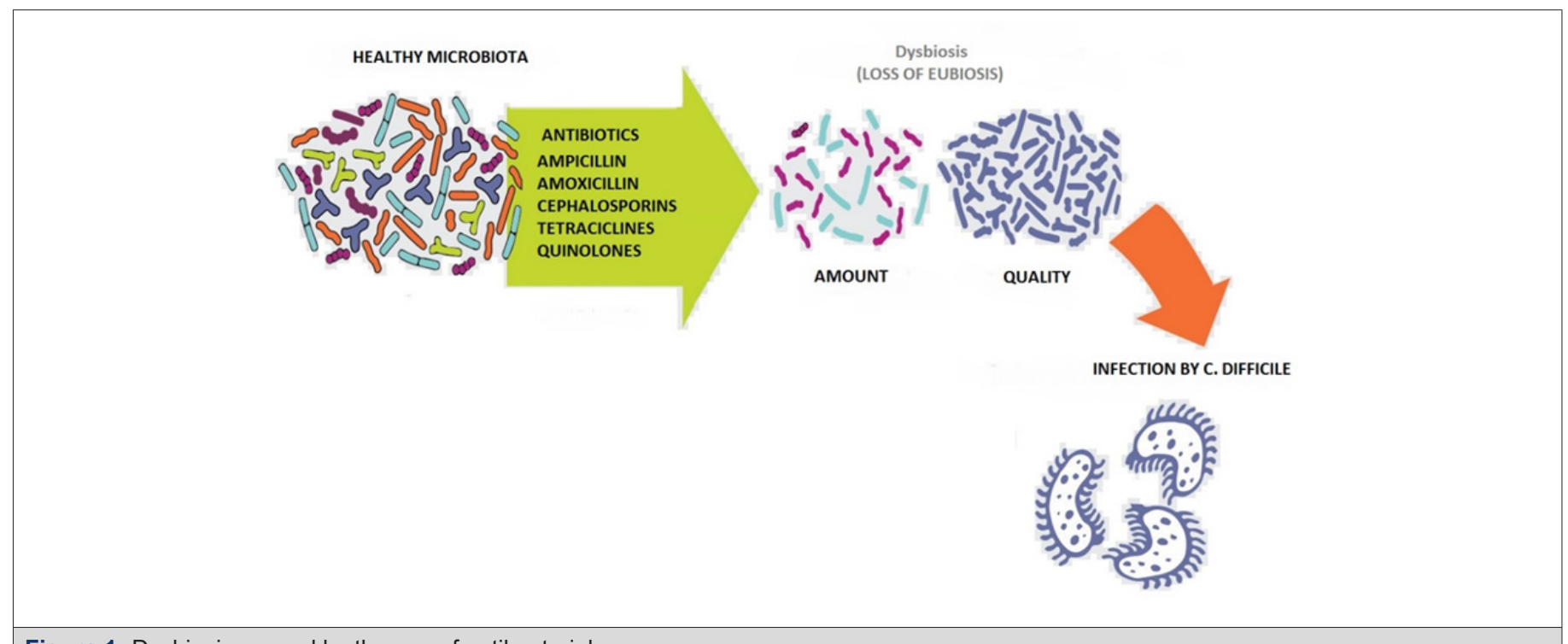

Figure 1: Dysbiosis caused by the use of antibacterials.

\section{Materials and Methods}

A narrative review was carried out, in which the PubMed, Scielo and ScienceDirect databases, among others, were searched. The collection and selection of articles was carried out in journals indexed in first and second languages from 2012 to 2020. As keywords, the following terms were used in the databases according to the DeCS and MeSH methodology: Clostridium difficile, Dysbiosis, Antibiotics In this review, 40 original and review publications related to the subject studied were identified, of which 15 articles met the specified inclusion requirements, such as articles that were in a range not less than the year 2012, which were articles of full text and to report on the pathogenicity mechanism of clostridium difficile and its association with dysbiosis caused by the use of antibacterials, as exclusion criteria it was taken into account that the articles did not have sufficient information and that they did not present the full text to the time of review.

\section{Results}

Antibiotics are often widely used today, in the face of gastrointestinal infections they play a very important role as a therapy to eradicate pathogenic microorganisms and their use can even be seen in prophylactic treatments, but many times their use can affect the health of the patients. people, causing unwanted adverse effects, diarrhea is one of the most common complications associated with the use of these, with most of these dysenteric pictures related to infections by c. difficille [4].
Historically, in the decade of the 70s, a significant number of diarrhea and colitis associated with the use of antibiotics to treat different infections of gastrointestinal origin began to appear, somehow it was found that in those patients who received treatment with clindamycin the picture was more noticeable. Studying this fact, it was found that around $21 \%$ of the patients receiving clindamycin developed diarrea [5], and around $10 \%$ of the patients presented a condition known as pseudomembranous colitis. This report was much commented on at the time due to the large number of cases of patients who received an antibiotic, and presented diarrhea and even colitis. Years later, with the development of medicine and the implementation of diagnostic tests, through stool samples that were saved from this epidemic, it was found that all presented the presence of cytotoxin B, associated with the pathogen Clostridium difficile. Later, it was observed that C. difficile was responsible for diarrhea in almost all patients receiving antibiotics, being more frequently found in patients receiving cephalosporin, ampicillin, and clindamycin [6].

At present, C. difficile infection can present asymptomatic or produce a marked pattern of diarrhea. Clinical signs can vary from mild or moderate diarrhea to pseudomembranous colitis, toxic megacolon, and even death. This disease is characterized by inflammation of the large intestine and the marked presence of plaques and accumulation of neutrophils in the lumen of the intestine [7]. There are several risk factors that have been associated with this disease, the most prominent and that has been mentioned 
on several occasions in this work is exposure to antimicrobials and hospitalization for a long period of time, but on the other side we also have advanced age, the severity of another underlying disease and immunosuppression, playing an important role in the course of the disease and the severity of it. Various studies have shown that the use of antacids and medications such as metformin could also be a risk factor [8].

The mechanism of pathogenicity of Clostridium difficile is mainly given by the presence of spores, which need to germinate in order to produce the disease, so the presence of the natural microbiota present in the intestine of all human beings means that C. difficile does not can germinate and stay in its spore form. It is there when the treatment with antimicrobials at the gastrointestinal level causes a dysbiosis that allows this bacterium to germinate, reproduce and trigger the disease. In healthy individuals, the spores cross the duodenum and reach the jejunum, where they manage to germinate due to the presence of bile. The germinated spores reach the ileum until they reach the cecum. The microbiota metabolizes the cholate derivatives and converts them to deoxycholate, thus preventing C. difficile from growing. However, when antimicrobial agents are used, the microbiota is altered and the components derived from cholate increase, which allows an increase in the germination of C. difficile spores $[9,10]$.

Once germinated, C. difficile produces two toxins, toxin $\mathrm{A}$ and toxin $\mathrm{B}$, in addition to a binary toxin. These toxins are responsible for the pathogenesis of $\mathrm{C}$. difficile, so only strains of $\mathrm{C}$. difficile that can produce at least 1 of these 2 toxins can cause disease. These toxins are encoded by two different genes (tcdA and tcdB) that are found at the same pathogenicity locus (PaLoc). At this locus there are also three more genes that have the function of regulating the production of these toxins (tcdC, tcdR and tcdE). The tcdE gene codes for a holin in charge of making pores in the cytoplasmic membrane so that toxins can be released, while the tcdC and tcdR genes are the negative and positive regulators, respectively, of the production of these toxins [11].

On the other hand, the binary toxin is not encoded in this locus, which is why it induces the formation of protrusions in the microtubules of the cells, which increases the adherence of the bacteria to the intestinal epithelium, this is how the infestation occurs. by $\mathrm{C}$. difficile when the natural microbiota is altered by the use of antibiotics [12].

\section{Discussion}

The results of various studies support what is stated in this document, about the existence of a relationship between inhospital treatment with antibiotics and the appearance of C. difficile infections, due to the fact that antibiotics alter the intestinal microbiota causing dysbiosis, facilitating the establishment of this infectious process, as we have discussed throughout this article, the incidence of community-acquired C. difficile infections is considerably low compared to confirmed positive cases in the hospital, it has been reported that community-acquired infection occurs in young patients, generally with immunosuppression and without previous antibiotic therapy [13]. Regarding the role that antibiotics play in this disease, previous studies suggest that the frequency and recurrence of infection due to the use of antibiotics is the same, regardless of whether it is treated with vancomycin or metronidazole, however, the risk of developing complications it is slightly higher if vancomycin is used.

One of the factors that could be influencing within the hospital area is the lack of isolation, any patient with suspected C. difficile infection should be placed in an individual room to avoid the spread of spores, therefore, if we group the use antibiotics, exposure and contact with the infectious agent, we will surely have a C. difficile infection [14]. In conclusion, the present work shows the pathogenicity mechanism of clostridium difficile associated with dysbiosis caused by the use of antibacterials. For which we suggest the prophylactic and concomitant use of probiotics together with antibiotic therapy to avoid intestinal dysbiosis and reduce the risk of developing C. difficile infection, especially in the hospital area. It should be noted that the use of probiotics in patients with established C. difficile infection is not recommended [15].

\section{References}

1. Rodríguez Pardo D, Mirelis B, Navarro F (2013) Infecciones producidas por Clostridium difficile. Enfermedades infecciosas y microbiologia clinica 31(4): 254-263.

2. Leffler D A, Lamont J T (2015) Clostridium difficile infection. New England Journal of Medicine 372(16): 1539-1548.

3. Czepiel J, Dróżdż M, Pituch H, Kuijper E J, Perucki W, et al. (2019) Clostridium difficile infection. European Journal of Clinical Microbiology \& Infectious Diseases 38(7): 1211-1221.

4. Lawson P A, Citron D M, Tyrrell K L, Finegold S M (2016) Reclassification of clostridium difficile as clostridioides difficile (Hall and O’Toole 1935) Prévot 1938. Anaerobe 40: 95-99.

5. DePestel D D, Aronoff D M (2013) Epidemiology of Clostridium difficile infection. Journal of pharmacy practice 26(5): 464-475.

6. Lessa F C, Mu Y, Bamberg W M, Beldavs Z G, Dumyati G K, et al. (2015) Burden of Clostridium difficile infection in the United States. New England Journal of Medicine 372(9): 825-834.

7. Lessa F C, Gould C V, Mc Donald L C (2012) Current status of Clostridium difficile infection epidemiology. Clinical Infectious Diseases 55(suppl_2): 65-70.

8. Seekatz A M, Young V B (2014) Clostridium difficile and the microbiota. The Journal of clinical investigation 124(10): 4182-4189.

9. Burke K E, Lamont J T (2014) Clostridium difficile infection: a worldwide disease. Gut and liver 8(1): 1-6.

10. Rupnik M, Janezic S (2016) An update on Clostridium difficile toxinotyping. Journal of clinical microbiology 54(1): 13-18. 
11. Crobach M J, Vernon JJ, Loo V G, Kong L Y, Péchiné S, et al. (2018) Understanding Clostridium difficile colonization. Clinical microbiology reviews: 31(2): e00021-17.

12. Khanna S, Pardi D S (2012) Clostridium difficile infection: new insights into management, In Mayo Clinic Proceedings 87(11): 1106-1117.

13. Marra A R, Perencevich EN, Nelson R E, Samore M, Khader K, et al. (2020) Incidence and outcomes associated with Clostridium difficile infections: a systematic review and meta-analysis, JAMA network open 3(1): e1917597-e1917597.

14. Carroll K C, Mizusawa M (2020) Laboratory Tests for the Diagnosis of Clostridium difficile. Clinics in colon and rectal surgery 33(2): 73-81.

15. Imwattana K, Knight D R, Kullin B, Collins D A, Putsathit P, et al. (2020) Antimicrobial resistance in Clostridium difficile ribotype 017. Expert review of anti-infective therap 18(1): 17-25. 